\title{
Revisión de la casuística sobre sismicidad inducida por producción y almacenamiento de hidrocarburos
}

\author{
Lucas Vadillo Fernández, Francisco Javier Fernández Naranjo, Virginia Rodríguez Gómez y \\ Julio López Gutiérrez \\ Instituto Geológico y Minero de España. Ríos Rosas 23, 28003 Madrid \\ I.vadillo@igme.es \\ fj.fernandez@igme.es \\ v.rodriguez@igme.es \\ j.lopezgu@igme.es
}

\begin{abstract}
RESUMEN
En este artículo se revisan los procesos de tensión que se producen en la corteza durante: la extracción de gas; en el almacenamiento de gas; en la inyección de fluidos (agua, $\mathrm{CO}_{2}$ ) para estimular la extracción de hidrocarburos convencionales (EOR); en la extracción de hidrocarburos no convencionales con inyección de agua a presión y fracturación hidráulica (fracking); y en la eliminación, mediante inyección, del agua salina (produced water) procedente de la extracción de hidrocarburos convencionales, así como del agua salina de retorno (flowback) de la fracturación hidráulica de la explotación de hidrocarburos no convencionales, ambas con más de $40000 \mathrm{mg} / \mathrm{L}$ de Sólidos Totales Disueltos (TDS). Asimismo se revisa la tipología de fallas más propensas a sufrir deslizamientos y la sismicidad relacionada con cada uno de los casos anteriormente descritos consecuencia del aumento de la presión de poro durante la inyección y la disminución de la presión de poro durante al extracción.
\end{abstract}

Palabras clave: falla, hidrocarburo, magnitud local $\left(\mathrm{M}_{\llcorner}\right)$, magnitud momento sísmico $(\mathrm{Mw})$, yacimiento

\section{A review of case histories of induced seismicity caused by hydrocarbon production and storage}

\begin{abstract}
In this article we review the stress-strain relationships that take place in the crust during some of the main hydrocarbon production and storage processes: gas extraction; water injection in wells to stimulate the extraction of oil (EOR); unconventional hydrocarbon production by hydraulic fracturing (fracking); disposal of wastewater (saline water) from the extraction of conventional and unconventional hydrocarbons such as saline water return (flowback) of hydraulic fracturing, both with TDS higher than $40000 \mathrm{mg} / \mathrm{L}$. In addition, the type of faults that are more likely to slip and the induced seismicity related to the production and extraction of hydrocarbons are analysed.
\end{abstract}

Key words: fault, field, hydrocarbon, local magnitude $\left(M_{L}\right)$, moment magnitude $(M w)$

\section{ABRIDGED ENGLISH VERSION}

\section{Introduction}

Induced seismicity is produced at subsurface depths in the vicinity of operation sites for: hydrocarbon recovery; water injection (for conventional hydrocarbon recovery, EOR, or for unconventional hydrocarbon 
recovery by means of hydraulic fracturing); injection of saline water (produced water) from conventional hydrocarbon recovery and returned saline water (flowback) from unconventional hydrocarbon recovery by means of hydraulic fracturing.

Induced seismicity occurring during fluid injection is related to changes in the stress strain relationship on poroelastic materials due to the increase of the pore pressure and the decrease of the effective pressure (Fig. 1) (Raleigh et al., 1976; Aduskin et al., 2000), giving rise to earthquakes of magnitude $M_{L} \leq 3$ (Grasso, 1992).

Induced seismicity also occurs during fluid extraction and is related to the progressive compaction of the deposits in poroelastic media. Adjustments in the reservoir are produced and these strengths are communicated with the upper layers of the deposit, resulting in the decrease of pore pressure (Fig. 2) in faults and earthquakes of $M_{L} \leq 5$ (Grasso, 1992).

The geometry of the fault plays a significant role in fault reactivation. Faults with dip angles close to $60^{\circ}$, without cohesion or with a very low cohesion value, are more prone to reactivation (Byerlee, 1978).

Several examples of induced seismicity in the vicinity of operation sites for the recovery and storage of hydrocarbons and saline water injection around the world are analyzed, focusing on the triggering processes.

\section{Induced seismicity caused by hydraulic fracturing}

Bowland Shale.

Unexpected seismic activity was observed near the shale gas exploration well Preese Hall (Bowland shale, Blackpool area, United Kingdom), coinciding with the period of hydraulic stimulation. Fifty seismic events were detected from March 2011 to May 2011 in this aseismic area. The greatest event, of magnitude ML 2.3, occurred on 1 April, 2011. At that moment the bottom hole pressure reached 68.2 MPa (Eisner et al., 2011). This seismic event led to the cessation of the drilling activities. Later on, a deformation of more than 0.5 inches $(12.7 \mathrm{~cm}$ ) in the wellbore casing was identified between depths of 2584 and $2633 \mathrm{~m}$ (Pater and Baisch, 2011).

Two hypotheses were suggested then by Green et al. (2012). The first of them was attributed to the injection of pressured water in the fault plane itself or, secondly, to the vicinity of the fault plane. The author strongly leans toward the first option.

The fault is $50^{\circ} \mathrm{N}$ in strike, having a $70^{\circ} \mathrm{W}$ dip angle. A $2.3 M_{L}$ magnitude earthquake might require a slip of up to $1 \mathrm{~cm}$ and a minimum rupture area of $10000 \mathrm{~m}^{2}$.

\section{Induced seismicity caused by saline water (wastewater) injection}

Oklahoma Oil Fields

The Oklahoma oil field (Oklahoma,USA), is an important oil field located within the complex of the Wilzetta fault system. Several conventional hydrocarbon reservoirs were structurally contained in the Wilzetta traps/ faults system. The injection of water type EOR and, principally, the disposal of saline water (wastewater) in the oil reservoirs is a common practice in the traps of the Wilzetta fault. Wellhead pressure increased in several steps, starting with 2 bar ( $0.2 \mathrm{MPa}$ ) in 2001, reaching a maximum pressure of 36 bar (3.6 MPa) in 2006, at a depressurized reservoir (below hydrostatic pressure) depleted by earlier hydrocarbon production, exceeding the volume of oil extracted (Keranen et al., 2013).

In November 2011, a sequence of more than 100 earthquakes occurred at the proximity to several injection wells. Two $M_{w}=5.0$ earthquakes followed by a $M_{w}=5.7$ earthquake in the next $24 \mathrm{~h}$, at a depth lower than $4 \mathrm{~km}$, caused damage in several buildings in the vicinity of the Wilzetta fault. Both Keranen et al. (2013) and Sumy et al. (2014) suggested that the seismic sequence was induced by wastewater injection into the oil reservoirs that were structurally contained inside the Wilzetta fault system.

Sumy et al. (2014), also suggested that the seismic sequence (including the largest earthquake $M=5.7$ ) could have been promoted at the proximity of the injection wells by a stress increase as low as 0.1 bar $(0.01$ $\mathrm{MPa}$ ) on a fault plane.

\section{Seismicity Induced by injection of wastewater from Enhanced oil recovery technologies (EOR)}

The Rangely Oil and Gas Field

The Rangely oil and gas field is located in Rio Blanco County (Colorado, USA), within the Rangely anticline structure. Gas and oil recovery has been carried out since 1945. The principal oil reservoir rocks are 
Vadillo Fernández, L., et al., 2017. Revisión de la casuística sobre sismicidad... Boletín Geológico y Minero, 128 (1): $241-252$

sandstones at a depth of about $1700 \mathrm{~m}$. Injection of water from EOR began in 1957 and finished in 1986, when the company started using $\mathrm{CO}_{2}$ instead of water for the EOR process (Raleigh et al., 1976).

A total of 976 earthquakes occurred between 1962 and 1970, 320 of them with a magnitude $M>1$. The greatest event, $M_{L} 3.4$ took place in 1964 (Gibbs et al., 1973).

A local network of seismographs was installed in 1969 in order to assess the relationship between the volume of the injected water and the seismicity. Between October 1969 and November 1970, pressure measured at the bottom hole of the reservoir reached 235-275 bar (23.5-27.5 MPa). During this period of time, a total of 900 earthquakes were registered (Raleigh et al., 1976).

The experiment showed a decrease in the seismic activity as water injection and pressure at the bottom hole declined (Raleigh et al., 1976; Gibbs et al., 1973).

\section{Seismicity Induced by hydrocarbon extraction}

NetherlandS On-Shore Gas Field

The Netherlands have 125 gas fields in production, including Groningenfield, the largest in Western Europe, in an area considered to be of low seismicity. About 350 earthquakes have been registered by the Royal Dutch Meteorological Institute (KNMI) the largest being a magnitude of 3.5 recorded in the field of Bergermeer (Van Eijs et al, 2006).

The first earthquake took place in 1986 in the Bergermeer field. Seismographs and accelerometers devices were installed, one of the last located at a depth of $200 \mathrm{~m}$.

A total of 606 earthquakes were registered between 1986 and 2009. The greatest of them, with $M=3.0$ and $M=3.5$ magnitude took place in the Bergermeer gas field. The epicentres were located at 2.2 and $2.5 \mathrm{~km}$ of depth, associated with normal faults having a dip angle that is prone to fault reactivation (Byerlee, 1978; Streit and Hillis, 2004; Mulders, 2003).

The subsidence observed since 1972 to 2006, was estimated to be $10.5 \mathrm{~cm}$, being the highest value of subsidence in this area, where the Bergen, Greet and Bergermeer fields intersect (TNO, 2008). Induced seismicity was interpreted to be related with the decrease of the pore pressure within fault plane, having a strike $\mathrm{N} 160^{\circ}$ and a dip angle between $53^{\circ}$ and $63^{\circ}$, which makes it prone to fault reactivation.

\section{Seismicity Induced by underground gas storage}

\section{Amposta/Castor Underground Gas Storage Facility}

The Castor Underground Storage Facility is a depleted offshore oil reservoir, located $20 \mathrm{~km}$ from the Mediterranean coast of Vinarós (Castellón, Spain).

It is located in the Amposta structure that consists of a structural horst faulted oil trap, in the western edge of the "Fosa del Surco de Valencia" geological unit (Trench of Valencia Groove) (Barat, 2011). The existence of some fault systems with interpreted neotectonic activity in this area has been found, such as the Eastern Amposta Fault and the Northern Montsia Fault. Gas injection in the Castor Underground Storage Facility began in 2013, at a depth of $1800 \mathrm{~m}$. Somehow a spatially localized seismic sequence started on 5 September, 2013. The greatest earthquakes were of magnitude $M w=3.6$ on 24 September, 2013, and Mw= 4.2 on 1 October, 2013, respectively (http://www.ign.es/ign/layout/n/sismoFormularioCatalogo.do).

Three possible scenarios were analysed by Cesca et al. (2014a) and Cesca et al. (2014b): 1) reactivation of the Eastern Amposta Fault; 2) a low-dip failure plane striking roughly parallel to the Eastern Amposta Fault but dipping perpendiculary to it; 3) a system of NW-SE almost-vertical faults (Montsia system). Cesca et al. (2014a) and Cesca et al. (2014b) concluded that scenarios 1 and 2 were feasible but excluded the reactivation of the Eastern Amposta Fault.

\section{Conclusions}

The analysed studies show a clear relationship between the changes that take place in the pore pressure of the saturated materials in faults acting as hydrocarbon seals/traps or in faults in contact with other fluids such as hydrocarbons, water, $\mathrm{CO}_{2}$, etc. These changes take place both during fluid injection and extraction. Fluid injection increases the pore pressure and subsequently it decreases the shear strength and finally causes the rupture of the materials affected by the fault. Fluid extraction leads to decompression, the readjustment of the coating materials and the decrease of the pore pressure in faults. Those faults dipping about $60^{\circ}$ are shown to be the most susceptible to slip. 
Vadillo Fernández, L., et al., 2017. Revisión de la casuística sobre sismicidad... Boletín Geológico y Minero, 128 (1): $241-252$

\section{Introducción}

La reciente actividad sísmica en zonas supuestamente estables, desde un punto de vista sismotectónico, o con una actividad sísmica moderada, ha dado lugar a una fuerte discusión acerca de la relación entre la presencia de estos sismos y la actividad industrial relacionada con la inyección y extracción de fluidos. La actividad sísmica originada por estas actividades recibe el nombre de sismicidad inducida.

La sismicidad inducida se produce en el entorno próximo, a profundidades subsuperficiales, durante las distintas tipologías de operaciones de la industria de hidrocarburos:

1) Durante la inyección de agua, bien para la recuperación de hidrocarburos convencionales (Enhanced Oil Recovey, EOR), o para la extracción de hidrocarburos no convencionales (Shale Gas, Oil Shale y Tight Oil) mediante fracturación hidráulica.

2) Durante la eliminación mediante inyección profunda del agua salina (produced water) procedente tanto de la extracción de hidrocarburos convencionales, como del agua salina del retorno (flowback) de las operaciones de fracturación hidráulica de hidrocarburos no convencionales, ambas con más de $40000 \mathrm{mg} / \mathrm{L}$ de Sólidos Totales Disueltos (TDS). Hay que considerar que a partir de, aproximadamente, $40000 \mathrm{mg} / \mathrm{L}$ de TDS la desalinización del agua salina para su reutilización, encarecen el proyecto (Acharya et al., 2011), por lo que en algunos estados, mayoritariamente, Texas, Ohio, Oklahoma, acaba inyectándose en sondeos profundos. La inyección mediante sondeos tipo EOR, como los de agua salina está regulada por el programa Underground Injection Control Programme de la U.S Environmental Protection Agency (E.P.A) como sondeos de Clase II.

3) En la extracción de gas.

4) En la inyección de gas para almacenamiento.

El objetivo de este artículo es realizar una revisión de los principales mecanismos de sismicidad asociados a procesos que se realizan durante las operaciones de extracción, almacenamiento de hidrocarburos e inyección de agua salina, con casos concretos ocurridos en diferentes partes del mundo.

\section{Cambios tensionales en la corteza por inyección y ex- tracción de fluidos}

En primer lugar se considera necesario distinguir entre sismicidad inducida (induced seismicity) y la sismicidad anticipada (triggered seismicity). La primera ocurre cuando se produce un cambio de esfuerzos sobre la falla que es comparable, en magnitud, al esfuerzo cortante de la falla, presentando niveles de sismicidad superiores a los esperables de acuerdo con el registro sísmico. La segunda se produce cuando con un pequeño cambio en el estado de esfuerzos de la falla se supera el esfuerzo cortante de la falla, acelerando el ciclo sísmico natural de ésta (McGarr and Simpsom, 2002). Zurich Risk Engeneering, define como sismo inducido (human-induced) aquel sismo que no habría ocurrido sin la acción humana y define como sismo anticipado (human-triggered) aquel sismo que habría ocurrido en cualquier momento pero ha sido anticipado (advanced) por la acción humana.

Ambos tipos de sismicidad pueden dar lugar a sismos cuyo momento sísmico depende entre otros parámetros de la superficie de ruptura y de la magnitud del desplazamiento. (Aki, 1966). La realización de operaciones de inyección a elevada presión en áreas cercanas a fallas se convierte en una potencial fuente de sismos. Las condiciones de deslizamiento en una falla se pueden caracterizar por el criterio de rotura de Mohr-Coulomb que relaciona la resistencia al corte requerido para el deslizamiento de la falla, la cohesión y el coeficiente de fricción en la falla, la tensión normal en el plano de falla y la presión del fluido de poro (Nicholson, 1951).

$$
\tau=\mathrm{c}+\sigma^{\prime} \operatorname{tg} \Phi ; \quad \sigma^{\prime}=\sigma-\mathrm{u}
$$

Por lo que:

$$
\tau=\mathrm{c}+(\sigma-\mathrm{u}) \operatorname{tg} \Phi \quad \text { [ecuación 1] }
$$

Siendo, $\tau$ la resistencia al corte; c la cohesión; $\sigma^{\prime}$ la tensión normal efectiva; $\sigma$ tensión normal total; u la presión de poro o presión intersticial y $\Phi$ el ángulo de rozamiento interno.

La sismicidad durante la inyección y extracción de fluidos está relacionada con cambios tensión-deformación en la proximidad de la falla, mediante variaciones de la presión de poro y de la presión efectiva (Raleigh et al., 1976; Aduskin et al., 2000), llegándose a producir sismos con $\mathrm{M}_{\mathrm{L}} \leq 3.0$ (Grasso, 1992).

Un comportamiento puramente teórico y sobresimplificado frente a una inyección implicaría un desplazamiento del círculo de Mohr en dirección a la envolvente de rotura mientras que la extracción lo haría en dirección contraria, manteniéndose uniformes los diámetros de los círculos de Mohr (Figura 1). No obstante este modelo teórico no resulta válido en casos reales debido a que no contempla efectos poroelásticos (Hillis, 2000).

La inyección o extracción no sólo implicaría, por tanto cambios en la magnitud de los esfuerzos máximo y mínimo y desplazamientos homogéneos del círculo de Mohr hacia la derecha e izquierda, sino que también podría implicar cambios en el diámetro del 


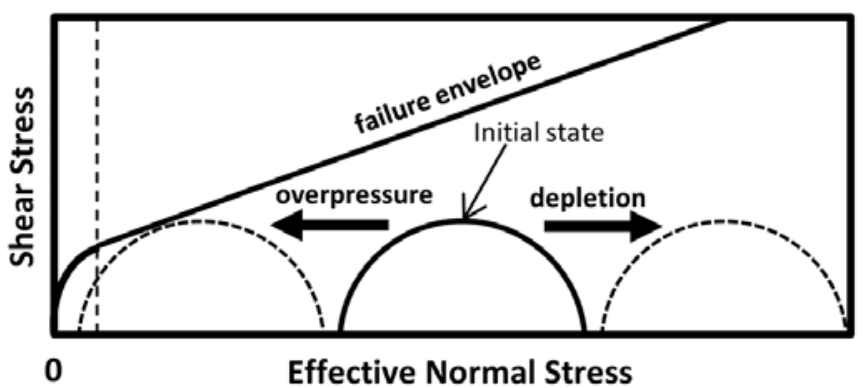

Figura 1. Diagrama sobresimplificado de Mohr-Coulomb mostrando los efectos teóricos del incremento de presión de poro (overpressure) y descenso de presión de poro (depletion), modificado de Hillis (2000).

Figure 1. Oversimplistic Morh Circle diagram showing theorical effects of increasing pore pressure (overpressure) and decreasing pore pressure (depletion). Modified from Hillis (2000).

círculo de Mohr de acuerdo a la teoría poroelástica (Streit and Hillis, 2004; Hillis, 2000; Nacht et al., 2010).

De acuerdo a las consideraciones poroelásticas realizadas por Hillis (2000), las diferencias existentes entre los gradientes de presión de poro para los parámetros $\sigma_{\mathrm{v}}^{\prime}$ y $\sigma_{\mathrm{h}}^{\prime}$ en los procesos de inyección $\mathrm{y}$ extracción (manteniéndose $\sigma_{v}$ constante en cualquier caso independientemente del gradiente de presión de poro) provocarían cambios respecto a los valores iniciales de $\sigma_{v}-\sigma_{h}$, lo que podría conllevar cambios en el valor del radio de los círculos de Mohr.

De esta manera, durante la inyección de fluidos (overpressure), el mecanismo primordial que controlaría el desarrollo de sismos sería el propio desplazamiento del círculo de Mohr en dirección a la envolvente de rotura, de manera que en el momento en que la resistencia al corte, alcance o supere la envolvente de rotura de esfuerzos totales se producirá un deslizamiento de falla y la consecuente sismicidad (Streit and Hillis, 2004; Nacht et al., 2010) (Figura 2).

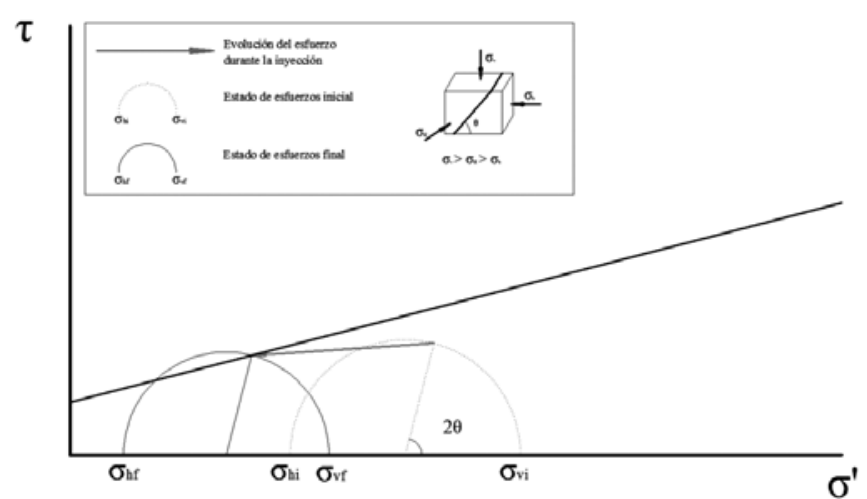

Figura 2. Diagrama de Mohr-Coulomb para una falla reactivada durante la inyección de fluidos. Modificado de Nacht et al. (2010).

Figure 2. Mohr-Coulomb diagram for a reactivated fault during injection.Modified from Nacht et al. (2010).
Para el caso de la extracción (depletion) se provocaría el desplazamiento teórico de los círculos de Mohr en dirección contraria a la envolvente de rotura, lo que se produce al experimentar $\sigma^{\prime}{ }_{\mathrm{h}}$ y $\sigma^{\prime}$ un incremento respecto del estado inicial de acuerdo a Hillis (2000). En este caso al ser dicho incremento mayor para el caso de $\sigma_{\mathrm{v}}^{\prime}$ respecto de $\sigma_{\mathrm{h}^{\prime}}$ gráficamente se traduciría en un aumento significativo del radio del círculo de Mohr, además del mencionado desplazamiento (Nacht et al., 2010), provocando que el círculo pueda llegar a intersectar con la envolvente de rotura (Figura 3).

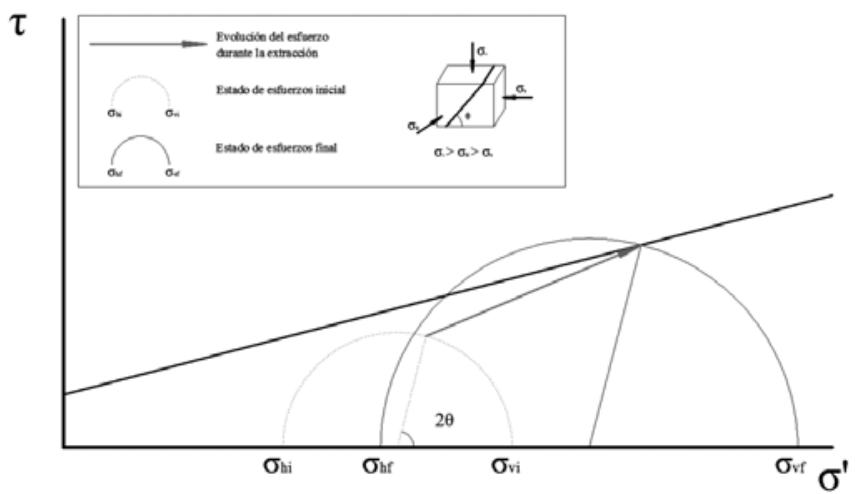

Figura 3. Diagrama de Mohr-Coulomb para una falla reactivada durante la extracción de fluidos. Modificado de Nacht et al. (2010).

Figure 3. Mohr-Coulomb diagram for a reactivated fault during depletion. Modified from Nacht et al. (2010).

En relación con este proceso, Grasso (1992) describe dos tipos de sismos: el primer tipo son sismos superficiales relacionados con la progresiva compactación del yacimiento, produciendo ajustes en el reservorio y comunicando estos esfuerzos a las capas superiores del yacimiento. La extracción de hidrocarburos da lugar a una disminución de la presión de poro en las fallas y el desplazamiento del círculo de Mohr hacia la derecha dando lugar a subsidencias con deslizamientos de falla y sismos de ML $\leq 5.0$; el segundo tipo de sismos está relacionado con la extracción masiva de hidrocarburos de grandes yacimientos con reajustes en la corteza terrestre debido a la redistribución de cargas en la parte cercana a la corteza dando lugar a terremotos $M L \geq 6.0$.

La geometría de las fallas juega un importante papel en la reactivación de éstas. Byerlee (1978) sostiene que las fallas con coeficientes de fricción $0.6 \leq \mu$ $\leq 1\left(31^{\circ} \leq \Phi \leq 45^{\circ}\right)$, con cohesión $\leq 1 \mathrm{MPa}$ y ángulo de buzamiento próximo a $60^{\circ}$ son las más propensas a la reactivación. 
Vadillo Fernández, L., et al., 2017. Revisión de la casuística sobre sismicidad... Boletín Geológico y Minero, 128 (1): $241-252$

En la misma línea, Streit and Hillis (2004) sostienen que, para una hipotética falla con coeficiente de rozamiento de 0.6 , sin cohesión o con cohesión 4 $\mathrm{MPa}$, una diferencia de presión de $20 \mathrm{MPa}$ a $2 \mathrm{~km}$ de profundidad durante la inyección es suficiente para reactivar fallas con buzamientos inferiores a $60-75^{\circ}$.

Así mismo, Mulders (2003), mediante la modelización de distintas geometrías de extracción de gas, llega a la misma conclusión fallas con ángulos de 60$70^{\circ}$ de buzamiento son más propensas a la reactivación, especialmente si la dirección del movimiento, durante la extracción, es similar a la dirección natural de la falla.

En los siguientes apartados se analizan diferentes casos de sismicidad inducida según los procesos desencadenantes.

\section{Sismicidad inducida por fracturación hidráulica}

\section{Bowland Shale}

Sin duda alguna es en la extracción de gas “no convencional" en margas, lutitas, limolitas (Shale gas) mediante fracturación hidráulica con agua a presión, donde se ha podido comprobar la existencia de fenómenos de sismicidad inducida debido a la monitorización de fracturas, in situ, de muchos de estos yacimientos. Uno de los casos que más repercusión mediática tuvo fue el yacimiento de shale gas de Bowland (Bowland Shale), en Blackpool (Gran Bretaña) donde, entre el 31 de marzo y el 27 de mayo de 2011, se detectaron 50 sismos, durante la fase de fracturación hidráulica. El mayor sismo, de $\mathrm{M}_{\mathrm{L}}=2.3$, profundidad $3.6 \mathrm{~km}$, se produjo el 1 de abril cuando la presión en el fondo del sondeo alcanzaba, aproximadamente, $9900 \mathrm{psi}$, equivalentes a $68.2 \mathrm{MPa}$ (Eisner et al., 2011), presión necesaria para abrir los planos de fractura de la pizarra con un alto gradiente de esfuerzos horizontales (mínimo de $0.75 \mathrm{psi} / \mathrm{ft}$ equivalente a $0.52 \mathrm{MPa}$ y máximo de $1.25 \mathrm{psi} / \mathrm{ft}$ equivalente a $0.86 \mathrm{MPa}$ ). Este sismo dio lugar a la paralización de las actividades en el sondeo de investigación. Posteriormente se comprobó que el tubo de acero (casing) del sondeo se había deformado más de 0.5 pulgadas $(12.7 \mathrm{~cm})$, a una profundidad de entre 2584 y 2633 m (Pater and Baisch, 2011). En Gran Bretaña se registran al año entre 300 y 400 sismos, de los que solo 40 tienen magnitudes mayores de $M_{L}$ 2.0 y únicamente 20 son sentidos por la población. Asimismo, el mayor terremoto del s. XX en el interior de Gran Bretaña ocurrió en 1984 en Lleyn (norte de Gales) tuvo una magnitud de $5.4 \mathrm{M}_{\mathrm{L}}$ y ocurrió a una profundidad de $22 \mathrm{~km}$ en la corteza inferior (McCue et al., 2007). De ello se deduce que Gran Bretaña es una típica zona con sismicidad baja, y por lo tanto un evento sentido por la población tiene bastante repercusión.

Se sugirieron dos posibles teorías, la primera que el sondeo inyectara directamente el agua a presión en la misma falla, la segunda que el sondeo inyectara agua a unos pocos cientos de metros de la falla, considerándose más plausible la primera de ellas. La falla tiene una orientación $50^{\circ} \mathrm{N}$, buzamiento $70^{\circ} \mathrm{O}$, pudiendo haberse producido un deslizamiento de falla de $1 \mathrm{~cm}$ y un área de ruptura de $10000 \mathrm{~m}^{2}$ (Green et al., 2012).

\section{Sismicidad inducida por inyección de agua salina}

\section{Oklahoma Oil Fields}

Una de las prácticas habituales en los EE.UU es la eliminación de agua salina que se extrae de yacimientos convencionales, así como del agua salina de retorno (flowback) proveniente de la fracturación hidráulica del petróleo y gas no convencional.

En el año 2011, existían en el Estado de Oklahoma 9639 sondeos tipo Class II, 5506 EOR y 4124 de agua salina (Murray and Holland, 2014). Un importante campo de hidrocarburos se encuentra en el sistema de fallas de Wilzetta Fault (Oklahoma) que se extiende a lo largo de $90 \mathrm{~km}$ en dirección NNE con buzamiento $80-85^{\circ}$ (Gay, 2003). El sistema de bloques-fallas constituye trampas de yacimientos de hidrocarburos convencionales, en los cuales se inyectó agua para la estimulación de hidrocarburos (EOR). Estas formaciones están constituidas por dolomías kársticas, areniscas, calizas y carbonatos del Cámbrico superior al Devónico Medio (Charpentier, 1995; Murray and Holland, 2014). Además se inyectaron grandes volúmenes de agua salina (produced water) en varias formaciones, principalmente en la formación de carbonatos Hunton (Ordovícico Superior-Devónico Inferior) y Arbuckle Group (Cámbrico superior-Ordovícico inferior) (Murray and Holland, 2014; Keranen et al., 2013).

La inyección de agua EOR en los yacimientos de petróleo y, principalmente, la eliminación del agua salina (produced water) es una práctica habitual en el sistema de trampas/fallas de Wilzetta Fault. El volumen de agua salina inyectada aumentó considerablemente en el año 2011, llegando en algunos puntos del sistema de trampas/fallas a inyectarse más de 500000 barriles por mes $\left(59620 \mathrm{~m}^{3}\right.$ ) (Murray and Holland, 2014). En algunos de estos sondeos despresurizados (presión por debajo de la presión 
Vadillo Fernández, L., et al., 2017. Revisión de la casuística sobre sismicidad... Boletín Geológico y Minero, 128 (1): $241-252$

hidrostática) se pasó de 2 bares ( $0.2 \mathrm{MPa})$ a 36 bares (3.6 MPa) (medidos en la cabeza del pozo) en cinco años, sobrepasándose el volumen de petróleo extraído (Keranen et al., 2013). El aumento de volumen y el aumento de presión se transmitieron al sistema de fallas/trampas, que se encuentran selladas y saturadas con el incremento de la presión de poro en el entorno de los planos de falla. En noviembre de 2011 se produjo una serie de más de cien sismos, con dos sismos de $M w=5.0$, seguido de otro de $M w=5.7$, en menos de 24 horas, a profundidades inferiores a 4 $\mathrm{km}$, causando diversos daños en edificios en pueblos del entorno de la falla. Tanto Keranen et al. (2013) como Sumy et al. (2014) sugieren que la serie de sismos fueron originados por la inyección de agua en el sistema de fallas de Wilzetta. Así mismo, Sumy et al. (2014) indican que dicha serie, incluido el sismo de $M w=5.7$, ocurrió dentro de las zonas de inyección en las que hubo un aumento de presión superior a 0.1 $\operatorname{bar}(0.01 \mathrm{MPa})$.

Al igual que ocurre en Gran Bretaña, el estado de Oklahoma se puede considerar de baja actividad sísmica natural. De acuerdo con los datos mostrados por el Leonard Geophysical Observatory, del Servicio Geológico de Oklahoma, (http://www.okgeosurvey1. gov/pages/earthquakes/faq.php), entre 1979 y 2008, el número anual de sismos con Mw superior a 3.0, en todo el Estado, fue siempre inferior a 1.0. Desde 2010 hasta la actualidad, el número se ha ido incrementando progresivamente hasta alcanzar los casi 200 en 2014, coincidiendo con el fomento de las actuaciones de inyección de aguas salinas. Existe una evidente relación causa efecto, siendo probablemente el incremento de la presión de poros, el mecanismo desencadenante.

\section{Sismicidad inducida por inyección de agua para la es- timulación y producción de petróleo (EOR)}

En los yacimientos convencionales de petróleo esté tiende a surgir de forma natural, a medida que el yacimiento va perdiendo energía el petróleo deja de fluir disminuyendo el ritmo de producción. Todavía se puede mantener la producción, generalmente, mediante técnicas de bombeo, etc. El factor de recuperación medio de esta recuperación primaria varía entre un $20-40 \%$. Para desplazar el petróleo y aumentar su producción se inyectan fluidos, generalmente, agua o gas $\left(\mathrm{CO}_{2}\right.$, Nitrógeno, etc.) mediante esta técnica se consigue extraer entre el 50 y $70 \%$ del petróleo. Esta técnica de recuperación secundaria recibe el termino Enhanced Oil Recovey (EOR) (Muggeridge et al., 2014).

\section{The Rangely Oil and gas field}

The Rangely Oil and gas field se encuentra situado en el anticlinal Rangely en el condado de Río Blanco, Colorado, USA. La extracción de gas y petróleo comenzó en 1945 prolongándose en el tiempo hasta la actualidad. El yacimiento se encuentra en unas areniscas a una profundidad de $1700 \mathrm{~m}$, tiene una porosidad del $12 \%$ y una permeabilidad $1 \mathrm{mDarcy}$. Debido a la baja permeabilidad del yacimiento, el caudal de los pozos declinó rápidamente una vez iniciadas las labores de extracción. En 1957 se comenzó a inyectar agua hasta 1986, momento en que la operadora cambio a $\mathrm{CO}_{2}$ para poder proceder a la recuperación el petróleo (Raleigh et al., 1976).

Entre 1962 y 1970 se produjeron 976 sismos, 320 con magnitud $M>1.0$, el mayor de $\mathrm{M}_{\mathrm{L}}=3.4$ en 1964 (Gibbs et al., 1973).

En 1966, el USA Geological Survey diseñó un experimento para controlar y determinar la fiabilidad de la generación de sismos colocando 4 sismógrafos sobre el reservorio a lo largo de dos áreas en una falla normal, de dirección NE-SO, que corta el eje de un anticlinal, donde se había inducido una elevada presión de poro. La máxima presión en el yacimiento se alcanzó en 1967 llegando a 290 bares (29 MPa) (Raleigh et al., 1976).

En 1969 se instalaron 14 sismógrafos adicionales en el yacimiento con el fin de demostrar la relación entre el volumen inyectado y la sismicidad inducida. La presión en el yacimiento se controló mediante cuatro sondeos. Entre octubre de 1969 y noviembre de 1970 las presiones en el fondo del yacimiento alcanzaron un valor de 235 a 275 bares ( 23.5 a 27.5 $\mathrm{MPa})$, tiempo durante el cual se produjeron 900 seísmos (Raleigh et al., 1976).

Se registró la actividad sísmica, detectándose epicentros en el sector suroeste de una falla, relacionando los periodos de inyección de agua y el incremento de la presión del yacimiento con la sismicidad inducida. El experimento demostró la disminución de la actividad sísmica a medida que se dejaba de inyectar agua y caía la presión en el fondo de los sondeos y viceversa (Raleigh et al., 1976; Gibbs et al., 1973).

\section{Romashkino oil field}

El yacimiento de petróleo de Romashkino se encuentra en la cuenca Volga-Ural (Rusia). Con una longitud máxima de $70 \mathrm{~km}$, es el más grande de Rusia. El yacimiento consiste en una sucesión de capas de areniscas y carbonatos de 10 a $30 \mathrm{~m}$ de potencia a una profundidad entre 1600 y $1800 \mathrm{~m}$. El principal reservorio 
Vadillo Fernández, L., et al., 2017. Revisión de la casuística sobre sismicidad... Boletín Geológico y Minero, 128 (1): $241-252$

tiene una permeabilidad de 200-420 mDarcy y una porosidad de $18-20 \%$ (Borisovich Turantaev et al., 2002). La producción comenzó en 1949 y se ha mantenido hasta la actualidad, extrayéndose más de 15 billones de barriles de petróleo $\left(15 * 10^{9}\right)$ (Adushkin et al., 2000). En 1954 comenzó a inyectarse agua para recuperar el petróleo; en 1976 el volumen de agua inyectado había alcanzado $2.13 * 10^{9} \mathrm{~m}^{3}$ excediendo el volumen de petróleo extraído en un $104,7 \%$ (Borisovich Turantaev et al., 2002). La presión del agua en el reservorio inicialmente estimada entre 16 y $18 \mathrm{MPa}$ llegó a alcanzar un máximo de $25 \mathrm{MPa}$ dando lugar a una serie de sismos de baja a moderada magnitud, siendo el máximo registrado de $M=4.0$, en el año 1982. En 1985 se instaló una serie de sismógrafos en el reservorio, registrándose en 1986 un sismo de $M=3.8$, y de $M=4.0$, en 1991. Gracias a ellos se pudo observar que la actividad sísmica aumentaba cuando el volumen de agua inyectada supera el volumen de petróleo extraído (Aduskin et al., 2000). Tanto la densidad de eventos sísmicos, como los pares de epicentros para periodos de menos de 24 horas (conforme a la metodología empleada, consistente en el agrupamiento de pares de eventos sísmicos por cercanía) correlacionaban positivamente la actividad sísmica con fallas de dirección NO-SE asociadas a la depresión tectónica que separa los campos de petróleo de Romashkino y Novoelokhovskoje y que disponían de saltos verticales de unos $50 \mathrm{~m}$ (Borisovich Turantaev et al., 2002).

\section{Sismicidad inducida por extracción de hidrocarburos}

\section{Netherland gas field on-shore}

Holanda tiene 125 yacimientos de gas en producción, incluido el de Groningenfield, el más grande de Europa Occidental en una zona considerada de baja sismicidad. Aproximadamente 350 sismos han sido registrados por Royal Dutch Meteorological Institute (KNMI) siendo el de magnitud más grande de 3.5 el registrado en el yacimiento de Bergermeer (Van Eijs et al., 2006).

El primer sismo en el yacimiento de gas de Bergermeer se produjo en 1986, instalándose a continuación una red de acelerómetros y sismógrafos, estos últimos instalados en sondeos de $200 \mathrm{~m}$ de profundidad (Mulders, 2003). Desde 1986 hasta 2009 se produjeron 505 sismos de $\mathrm{M}_{\mathrm{L}} \leq 1.0$, con un ratio anual de 30-50 sismos en los yacimientos de gas holandeses (Kraaijpoel et al., 2009). Todos los sismos están relacionados con caídas de presión de poro entre 122 y 287 bares (12.2 y $28.7 \mathrm{MPa}$ ) (Van Eijs et al., 2006)
En 1994 y 2001 se produjeron dos sismos de magnitud $\mathrm{M}_{\mathrm{L}}=3.0$ y $\mathrm{M}_{\mathrm{L}}=3.5$, respectivamente, cuyos epicentros se localizaron en el yacimiento de gas de Bergermeer (Hager and Nafi Toksöz, 2009). El yacimiento se ubica en una formación de areniscas con una porosidad media de un $23 \%$ teniendo a techo una formación de evaporitas que actúa de sello, constituyendo estructuralmente un sistema de horst limitado por dos fallas normales de direcciones y buzamientos $160^{\circ} / 53-62^{\circ}, 143^{\circ} / 68^{\circ}$ y una falla normal interna menor $160^{\circ} / 53-63^{\circ}$ que divide el yacimiento en dos bloques (TNO, 2008). Los epicentros se localizaron a 2.2 y $2.5 \mathrm{~km}$ de profundidad asociados a la falla normal interna, que presenta un ángulo de buzamiento propenso a la reactivación de la falla (Byerlee, 1978; Streit and Hillis, 2004; Mulders, 2003). La subsidencia observada entre 1972-2006 se estimó en $10.5 \mathrm{~cm}$, la máxima subsidencia se localizó en la zona noreste en la zona de confluencia de los yacimientos de Bergen, Greet y Bergermmer fields (TNO, 2008). La sismicidad inducida se relacionó con la disminución de la presión de poros en la falla interna menor, 160\%/53$63^{\circ}$, más propensa a la reactivación. El sismo de $\mathrm{M}_{\mathrm{L}}=3$, de 1994, se produjo después de una caída de la presión de 168 bares (16.8 $\mathrm{MPa}$ ) en el yacimiento (Van Eijs et al., 2006).

Van Eijs et al. (2006) identifican tres parámetros claves para determinar a priori si un yacimiento es susceptible o no de provocar sismicidad inducida como consecuencia de la producción de gas: la caída de presión en el yacimiento causada por la producción de hidrocarburos, la densidad de fallas existentes en el yacimiento y la diferencia de rigidez de las rocas sello y almacén del yacimiento.

Estos autores concluyen que la sismicidad inducida por la extracción de hidrocarburos de un yacimiento solo es posible si se dan, simultáneamente, las siguientes condiciones: caída de presión $(P)$ superior a la presión crítica, considerándose 72 bares (7.2 $\mathrm{MPa}$ ) como la caída de presión crítica por debajo de la cual no se produce sismicidad inducida $(\mathrm{P}>\mathrm{Pcrítica}=$ 72 bares = $7.2 \mathrm{MPa}$ ); un factor de densidad de fallas (F) superior al factor de densidad crítica, considerada de $0.93+0.03(F>$ Fcrítica $=0.93+0.03)$; un factor de rigidez (S) superior al factor crítico, considerado 0.98 +0.16 (S > Scrítica $=0.98+0.16)$.

\section{Lacq gas field}

El yacimiento de petróleo de Lacq (Francia) se encuentra en la cuenca de Aquitania, separada $25 \mathrm{~km}$ de la zona tectónica del frente norpirenaico. El yacimiento de petróleo se descubrió en 1949 mediante 
prospección sísmica, en las calizas fisuradas del Campaniense-Santoniense (Cretácico Superior), denominado Lacq Superior, a una profundidad media de $650 \mathrm{~m}$. En 1951 se descubrió gas condensado en dolomías del Pliensbachiense (Jurásico Inferior), de $500 \mathrm{~m}$ de potencia, y a $3500 \mathrm{~m}$ de profundidad, denominada Lacq inferior. Aunque la cuenca de Aquitania se encuentra, relativamente, cercana a la zona activamente sísmica de los Pirineos, hasta 1969 no se había localizado ningún epicentro en la región de Lacq (Rothé, 1977; Segall et al., 1994). La extracción de gas de Lacq sigue un modelo de deformación, compresivo en la parte central del yacimiento y extensivo en la parte exterior del yacimiento, con fallas normales en los flancos y fallas inversas en el yacimiento (Segall, 1989; Odonne et al., 1999).

En 1957 se dio comienzo a la explotación de dicho gas con una presión de poro en el yacimiento de 64 $\mathrm{MPa}$, en 1967 la caída de presión fue de $30 \mathrm{MPa}$ dando lugar a una subsidencia de $2.5 \mathrm{~cm}$ Entre 1967 y 1989 se produce una nueva caída de presión de $25 \mathrm{MPa}$ y una subsidencia añadida de $3 \mathrm{~cm}$ (Maury et al., 1991).

Los sismos dieron comienzo en 1969, coincidiendo con la caída de presión de $30 \mathrm{MPa}$, en 1972 se produjeron dos sismos con una magnitud estimada entre 3.0 y 4.0. En 1974 se instaló una red de geófonos y sismógrafos, registrando más de 1000 sismos durante el periodo 1974-1997, cuatro de ellos con magnitud superior a 4.0 , el $90 \%$ de los epicentros se localizaron encima del yacimiento a profundidades entre 2-7 km (Maury et al., 1991). La extracción de los dos campos de explotación (petróleo y gas) supuso una progresiva caída de presión de $55 \mathrm{MPa}$ a pesar de que durante la extracción de petróleo se estuvo inyectando el agua de producción, y a partir de 1975 se inyectaron $3.3 * 10^{6}$ metros cúbicos de agua de producción en los niveles inferiores del yacimiento de gas para intentar evitar la caída de presión, la consiguiente subsidencia y la deformación del yacimiento (Segall, 1989)

\section{Ekofisk Oil field}

El reservorio Ekofisk se encuentra situado en Central Graben, al sur del Mar del Norte, a 3200 m bajo el nivel del mar. La formación productiva tiene forma de anticlinal y consiste en dos niveles de caliza creta (chalk) fracturada: Formación TOR (Cretácico Superior) y Formacion Ekofisk (Paleoceno). El recubrimiento tiene una potencia aproximada de 3000 $\mathrm{m}$ de argilitas y lutitas. La estructura ha sido afectada por numerosos episodios tectónicos que han fracturado el reservorio facilitando la extracción de hidrocarburos y permitiendo la migración de gas a la formación Marker (Mioceno inferior) (Larsen, 2007).

La producción de petróleo de Ekofisk comenzó en el año 1971, durante 30 años de extracción de petróleo la presión cayó de $49.6 \mathrm{MPa}$ a $22.0 \mathrm{MPa}$ con una reducción de la porosidad del $38 \%$ a $33 \%$ y la consiguiente disminución de la presión de poro (Larsen, 2007; DoornHorf et al., 2006). Durante la extracción de hidrocarburos y la fase de compactación se produjo una subsidencia que alcanzó $8.26 \mathrm{~m}$ en algunos puntos del reservorio con una tasa de $0.40 \mathrm{~m} /$ año (Ottemoller et al., 2005). En 1984 las plataformas de Ekofisk habían sufrido las consecuencias de la subsidencia con la consiguiente deformación de los sondeos siendo necesario remplazar el complejo de plataformas de extracción con un coste de 3 billones dólares U.S. (DoornHorf et al., 2006).

En 1987 se empezó a inyectar agua para represurizar el reservorio disminuyendo el grado de subsidencia a 0.10-0.15 m/año. En 2001 se produjo un sismo de Mw entre 4.1-4.4. En 2002, se observaron altas presiones entre 1800 y $2100 \mathrm{~m}$ en el flanco norte del yacimiento y la deformación del casing de los sondeos confirmando el colapso del recubrimiento (Larsen, 2007).

Una batimetría de alta resolución indicó una subsidencia de $40 \mathrm{~cm}$ en la zona sur mientras la zona norte del yacimiento se había levantado $25 \mathrm{~cm}$. Se consideró que este levantamiento fue provocado por la rotura del recubrimiento al superar la presión de inyección la presión calculada con el test del leak-off (LOT) (Ottemoller et al., 2005; Teufel, 1996).

La rotura del sello dio lugar a que el agua de la inyección accediera a las fallas del recubrimiento, con dos posibles planos, una falla subvertical o una falla subhorizontal, produciendo un deslizamiento debido al aumento de la presión de poro durante la represurización (Ottemoller et al., 2005).

\section{Sismicidad inducida por almacenamiento de gas}

\section{Almacén subterráneo Amposta/Castor}

El Almacén de gas Castor es un antiguo campo petrolífero situado en el Mediterráneo, a unos $20 \mathrm{~km}$ de la costa de Vinarós (Castellón), bajo una lámina de agua de $60 \mathrm{~m}$. El yacimiento de petróleo fue explotado durante los años de 1973-1989 extrayéndose, aproximadamente, 53 millones de $\mathrm{m}^{3}$.

La estructura Amposta está constituida por una trampa tipo horst en el borde occidental del Dominio Geológico del Surco de Valencia, una estructura tipo rift de corteza continental adelgazada. El horst, con buzamiento de $20^{\circ} \mathrm{SE}$, constituye una trampa 
Vadillo Fernández, L., et al., 2017. Revisión de la casuística sobre sismicidad... Boletín Geológico y Minero, 128 (1): $241-252$

estructural cuyo cierre por el noroeste consiste en una falla con un salto de $1000 \mathrm{~m}$, cuyo bloque hundido está formado por materiales arcillosos de los grupos litoestratigráficos Castellón y Ebro. El resto de los cierres de la estructura los proporcionan los materiales de la Formación Lutitas del Grupo Castellón (Barat, 2011). El sello superior lo constituyen las lutitas, limolitas y areniscas arcillosas de la formación Castellón. El almacén está formado por unas calizas micríticas kársticas del Cretácico inferior (Barremiense) o calizas de Montsiá (Barat, 2011).

El margen occidental de la fosa de Valencia se caracteriza por ser una zona de sismicidad baja a moderada. En este sector se ha identificado la presencia de fallas con actividad neotectónica, entre otras, las fallas de Amposta Oriental y Montsiá Norte (IGME and ENRESA, 1998; IGME, 2012). La primera es una falla normal lístrica de dirección NNE-SSO, buzamiento $42^{\circ} \mathrm{O}$ en la parte superior y $17^{\circ} \mathrm{O}$ en la parte inferior; mientras que la segunda es una falla normal lístrica de dirección NNO-SSE y buzamiento $60^{\circ}$ en su tramo superior y $49^{\circ}$ en el inferior, hacia el OSO (Fernández et al., 2014).

En el año 2013 comenzó a inyectarse gas colchón en el denominado "Almacenamiento subterráneo Castor" a una profundidad aproximada de $1800 \mathrm{~m}$ (Barat, 2011). De acuerdo con el catálogo de datos del IGN el 5 de septiembre se inicia la actividad sísmica con dos series: una primera que comienza el 5 de septiembre alcanzándose, el 24 de septiembre, Mw= 3.6; y una segunda que comienza el 29 de septiembre dando lugar a varios seísmos, el mayor $\mathrm{Mw}=4.2$, el 1 de octubre (http://www.ign.es/ign/layoutln/sismoFormularioCatalogo.do). El clúster de epicentros se sitúa en las inmediaciones de la falla de Amposta Oriental y Montsiá Norte.

Mediante un estudio de inversión del tensor del momento sísmico, se estudiaron tres posibles escenarios (Cesca et al, 2014a; Cesca et al, 2014b): reactivación de la Falla de Amposta Oriental; reactivación de una falla menor de dirección paralela a la anterior y buzamiento SE; y reactivación de una o varias fallas perpendiculares a la falla de Amposta situada al NW, conocida como falla de Montsiá. Las conclusiones de este estudio determinan como escenarios plausibles el segundo y el tercero, descartando el escenario de reactivación de la Falla de Amposta Oriental (Cesca et al, 2014a; Cesca et al, 2014b).

\section{Conclusiones}

Los estudios revisados muestran una clara interrelación entre los cambios producidos en la presión de poro en materiales saturados situados en zonas/ planos de falla que constituyen sellos/trampa de hidrocarburos o fallas que están en contacto con hidrocarburos, agua, $\mathrm{CO}_{2}$, etc., respecto a fenómenos de sismicidad, bien durante la inyección de fluidos como durante la extracción. La inyección da lugar a un aumento de la presión de poro, a la disminución de la resistencia al corte y a la rotura de los materiales de la falla. La extracción da lugar a la descompresión y reajuste de los materiales del recubrimiento y la disminución de la presión de poro en las fallas. Además, se observa que las fallas en torno a los $60^{\circ}$ de buzamiento son las más propensas a sufrir deslizamientos.

Se han referenciado asimismo situaciones en las que la sismicidad está ligada a la inyección en yacimientos de petróleo agotados cuando el volumen de agua inyectada supera el volumen de petróleo extraído.

Las magnitudes de los sismos inducidos por reactivación de fallas preexistentes a las operaciones de extracción e inyección documentadas, superan la denominación de "microsismos", por lo que es necesario diferenciarlos de aquellos sismos de magnitud menor de $\mathrm{Mw}=2.0$, que se producen durante la fracturación de la roca en las operaciones de fracturación hidráulica y que normalmente no son sentidos por la población, o no pasan de causar una incómoda alarma. A diferencia de éstos últimos, la reactivación de fallas previas pueden generar terremotos que superen las magnitudes $\mathrm{Mw}=4.0$ y 5.0 , pudiendo provocar daños. Por ello que se precisa un estricto conocimiento estructural del medio subterráneo, y de la historia neotectónica de las fallas que se pueden ver afectadas. Un moderado incremento de la presión de poro, dependiendo del estado natural de las tensiones en el plano de falla, puede estimular el deslizamiento.

\section{Referencias}

Acharya, H.R., Henderson, C., Matis, H., Kommepalli, H., Moore, B. and Wang, H. 2011. Cost Effective Recovery of Low-TDS Frac Flowback Water for Re-use. United States Department of Energy D.C, 31 pp.

Adushkin, V.V., Rodionov, V.N., Turuntaev, S. and Yudin, A.E 2000. Seismicity in the oilfield. Oilfield Review, 12 (2), 2-17.

Aki, K. 1996. 4. Generation and propagation of $G$ waves from the Niigata earthquake of June 14, 1964. Part 2. Estimation of earthquake moment, released energy and stress-strain drop from $\mathrm{G}$ wave spectrum. Bulletin of the Earthquake Research Institute 44, 73-88.

Barat, C. 2011. Proyecto Castor de Almacenamiento Subterráneo de Gas Natural. Tierra y tecnología, 39, 3-8. 
Borisovich Turantaiev, S. and Andreevna Razumnaya, O. 2002. An Application of Induced Seismicity Data Analysis for Detection of Spatial Structures and Temporal Regimes of Deformation Processes in Hydrocarbon Fields. Pure and Applied Geophysics, 159, 421-447.

Byerlee, J.D. 1978. Friction of rocks. Pure Applied Geophysics, 116, 615-626.

Cesca, S., Grigoli, F., Heimann, S., González, A., Buforn, E., Maghsoudi, S., Blanch, E. and Dahm, T. 2014a. The seismic sequence related to the gas injection of the Castor Project offshore Spain. Una aproximación multidisciplinar al estudio de las fallas activas, los terremotos y el riesgo sísmico. Segunda Reunión Ibérica Sobre Fallas Activas y Paleosismología, Iberfault, Murcia, España, 217-220.

Cesca, S., Grigoli, F., Heimann, S., González, A., Buforn, E., Maghsoudi, S., Blanch, E. y Dahm, T. 2014b. The 2013 September-October seismic sequence offshore Spain: a case of seismicity triggered by gas injection? Geophysical Journal International, 198, 941-953.

Charpentier, R. 1995. Nemaha Uplift Province (055). U.S. Geological Survey. Digital Data Series DDS-30, Release 2, one CD-ROM.

Doornhof, D., Kristiansen, T.G., Nagel, N.B., Pattillo, P.D. and Sayers, C. 2006. Oilfield Review, 68 pp.

Eisner, L., Janska, E. and Matousek, P. 2011. Seismic analysis of the events in the vicinity of the Preese Hall well. Seismik report for Cuadrilla Resources, $28 \mathrm{pp}$.

Fernández, F., Bohoyo, F., Maestro, A. and GarcíaMayordomo, J. 2014. Nuevas aportaciones sobre las características geométricas del sistema de fallas Amposta Oriental-Montsià Norte (Margen Catalano-Valenciano, España). Una aproximación multidisciplinar al estudio de las fallas activas, los terremotos y el riesgo sísmico. Segunda Reunión Ibérica Sobre Fallas Activas y Paleosismología, Iberfault, Murcia, España, 105-108.

Gay, S.P. 2003. The Nemaha Trend-A system of compressional thrust-fold, strike-slip structural features in Kansas and Oklahoma, (Part 2, conclusion). The Shale Shaker. The Journal of the Oklahoma City Geological Society, 54 (2), 39-49.

Gibbs, J.F., Healy, J.H., Raleing, C. B. and Coakley, J. 1973. Seismicity in the Rangely, Colorado, Area: 1962-1970. Bulletin of the Seismological Society of America, 63 (5), 1557-1570.

Grasso, J. R. 1992. Mechanics of seismic instabilities induced by the recovery of hydrocarbons. Pure and Applied Geophysics, 139 (3-4), 507-534.

Green, C. A., Styles, P. and Baptie, B. J. 2012. Preese Hall shale gas fracturing. Review and recommendations for induced seismic mitigation. Department of Energy and Climate Change. GOV. UK, 26 pp.

Hager, B.H. and Nafi Toksöz, M. 2009. Technical review of Bergermeer seismicity study. TNO REPORT 2008-U-R1071/B, 34 pp.

Hillis, R. 2000. Pore pressure/stress coupling and its limitations for seismicity. Exploration Geophysics, 31, 448-454
IGME and ENRESA (1998) Mapas Neotectónico y Sismotectónico de España a escala 1:1000000. Vol. I (237 pp.) y Vol. II (167 pp.).

IGME 21/07/14. QAFI: Quaternary Active Faults Database of Iberia. http://info.igme.es/qafi/

IGN. 21/07/14. http://www.ign.es/ign/layoutln/sismoFormularioCatalogo.do.

Kraaijpoel, D., Goutbeek, F., Sleeman, R. and Dost B. 2009. Induced seismicity in the gas reservoirs of the Netherlands. Poster: EGU General Assembly 2009, April 20-24, 2009, Vienna.

Keranen, K. M., Savage, H.M., Abers, G. A. and Cochran. E. S. 2013. Potentially induced earthquakes in Oklahoma, USA: Links between wastewater injection and the $2011 \mathrm{Mw} 5.7$ earthquake sequence. The Geological Society of America, 41 (6), 699-702.

Larsen, A. 2007. Gas in overburden on Ekofisk. Department of Petroleum technology Institute of Technology and Science University of Stavanger, 89 pp.

Leonard Geophysical Observatory. Oklahoma Geological Survey. 29/06/15. http://www.okgeosurvey1.gov/pages/earthquakes/faq.php.

Maury, V.M.R., Grasso, J.R y Wittlinger, G. 1991. Monitoring of subsidence and induced seismicity in the Lacq Gas Field (France): the consequences on gas production and field operation. Engineering Geology, 32, 123-135.

McCue K.F., Musson, R.M.W. and Gibson G. 2007, A comparison of the seismicity of the UK and southeastern Australia, in AEES2007, The Australian Earthquake Engineering Society Conference, Wollongong NSW, 23 - 25 November 2007.

McGarr, A. and Simpson, A. 2002. 40 Case histories of induced and triggered seismicity. International Handbook of Earthquake and Engineering seismology. Volume 81A, 647-661.

Muggeridge, A., Cockin, A., Webb, K., Frampton, H., Collins, I., Moulds, T. and Salino, P. 2014. Recovery rates, enhanced oil recovery and technological limits. Philosophical Transactions of The Royal Society A-Mathematical Physical and Engineering Sciences, 372, 20120320. Doi: 10.1098/rsta.2012.0320.

Mulders, F.M.M. 2003. Modelling of stress development and fault slip in and around a producing gas reservoir. PhD thesis, Delft University Press, 272 pp.

Murray, K.E. and Holland, A. 2014. Structural Analysis of the Boktukola Syncline, Central Ouachita Mountains, Oklahoma; Inventory of Class II Underground Injection Control Volumes in the Mid-Continent. The Shale Shaker. The Journal of the Oklahoma City Geological Society, 65 (2), 98-106.

Natch, P. K., Oliviera, M., Roch, D.M. and Costa, A.M. 2010. Investigation of geological fault reactivation and opening. Mecanica Computacional, XXIX, 8687-8697.

Nicholson, C. and Wesson, R. L. 1951. Earthquake Hazard Associated with Deep Well Injection-A report to de U.S Environmental Protection Agency (EPA). U.S Geological Survey Bulletin, 74 pp. 
Odonne, F., Menard, I., Massonat, G. and Rolando, J. P. 1999. Abnormal reverse faulting above a depleting reservoir. Geology, 27, 111-114.

Ottemöller, L., Nielsen, H.H., Atakan, K., Braunmiller, J. and Havskov, J. 2005. The 7 May 2001 induced seismic event in the Ekofisk oil field, North Sea. Journal of Geophysical Research, 110, B10301. Doi: 10.1029/2004JB003374.

Pater, C.J. and Baisch, S. 2011. Geomechanical study of Bowland shale seismicity. Synthesis report. Cuadrilla Resources Ltd, $57 \mathrm{pp}$.

Raleigh, C. B., Healy, J. H. and Bredehoeft, J. D. 1976. An experiment in earthquake control at Rangely, Colorado. Science, 191, 1230-1237. Doi:10.1126/ science.191.4233.1230.

Rothé, J.P. 1977. Séismes artificiels et exploitations pétrolières I'exemple de Lacq (France). Tectonophysics, 9, 215-218.

Segall, P., 1989, Earthquakes triggered by fluide extraction: Geology, 17: 942-946.

Segall, P., Grasso, J.R. and Mossop, A. 1994. Poroelastic stressing and induced seismicity near the Lacq gas field, southwestern France. Journal of Geophysical Research, 99 (B8), 15423-15438.

Streit, J.E. and Hillis, R.R. 2004. Estimating fault stability and sustainable fluid pressures for underground storage of $\mathrm{CO}_{2}$ in porous rock. Energy, 29, 1445-1456.

Sumy, D. F., Cochran, E.S., Keranen, K.M., Wel, M. and Abers, G.A. 2014. Observations of static Coulomb stress triggering of the November 2011 M 5.7 Oklahoma earthquake sequence. Journal of Geophysical Research: Solid Earth, 119 (3), 1904-1923.

Teufel, L.W. 1996. Influence of Pore Pressure and Production-Induced Changes in Pore Pressure on In Situ Stress. Sandia National Laboratories, 54 pp.

TNO (Dutch Organization for Applied Scientific Research). 2008. Technical Review of Bergermeer Seismicity Study. TNO Report, 2008- UR1071/B, 95 pp.

Van Eijs, R.M.H.E., Mulders, F.M.M., Nepveu, M., Kenter, C.J. and Scheffers, B.C. (2006). Correlation between hydrocarbon reservoir properties and induced seismicity in the Netherlands. Engineering Geology, 84, 99-111.

Recibido: julio 2015

Revisado: enero 2016

Aceptado: octubre 2016

Publicado: marzo 2017 